\title{
Electric manipulation of electron spin relaxation induced by confined phonons in nanowire-based double quantum dots
}

\author{
M. Wang, ${ }^{1,2}$ Y. Yin, ${ }^{1}$ and M. W. $\mathrm{Wu}^{1,2, \text { ॠ }}$ \\ ${ }^{1}$ Hefei National Laboratory for Physical Sciences at Microscale, \\ University of Science and Technology of China, Hefei, Anhui, 230026, China \\ ${ }^{2}$ Department of Physics, University of Science and Technology of China, Hefei, Anhui, 230026, China
}

(Dated: November 8, 2018)

\begin{abstract}
We investigate theoretically the electron spin relaxation in single-electron nanowire-based semiconductor double quantum dots induced by confined phonons and find that the electron spin relaxation rate can be efficiently manipulated by external electric fields in such system. An anti-crossing, due to the coaction of the electric field, the magnetic field and the spin-orbit coupling, exists between the lowest two excited states. Both energies and spins of the electron states can be efficiently tuned by the electric field around the anti-crossing point. Multiple sharp peaks exist in the electric-field dependence of the spin relaxation rate induced by the confined phonons, which can be ascribed to the large density of states of the confined phonons at the van Hove singularities. This feature suggests that the nanowire-based double quantum dots can be used as electric tunable on-and-off spin switches, which are more sensitive and flexible than the ones based on quantum-well based double quantum dots. The temperature dependence of the spin relaxation rate at the anti-crossing point are calculated and a smooth peak, indicating the importance of the contribution of the off-diagonal elements of the density matrix to the spin relaxation, is observed.
\end{abstract}

PACS numbers: 72.25.Rb, 73.21.La, 63.22.-m, 63.20.kd

\section{INTRODUCTION}

Manipulation of spin relaxation in semiconductor quantum dots (QDs) is of great interest due to its potential applications in quantum information processing and spintronic devices. ${ }^{1-4}$ Various mechanisms can lead to the spin relaxation, such as the spin-orbit coupling (SOC) due to the space inversion asymmetry together with the electron-phonon scattering,,$-\frac{5}{-8}$ the coaction of the hyperfine interaction and the electron-phonon scattering, ,5,9-11 the g-factor fluctuations and the direct spin-phonon scattering due to the phonon-induced strain.$^{5}$ For the widelyinvestigated III-V semiconductor QDs, the SOC together with the electron-phonon scattering dominates the spin relaxation in large magnetic fields.$^{6-8,12,13}$ It has been shown that the spin relaxation can be manipulated not only in single QDs by external magnetic field, $, 14,15$ but also in double quantum dots (DQDs) by external electric field ${ }^{16,17}$ The latter is more interesting since the electric field is more easily accessible and controllable in genuine devices. The electric coherent spin manipulation in DQDs has been demonstrated experimentally by electric dipole spin resonance with weak electric field ${ }^{18,19}$ It was also suggested that the electron spin in DQDs can be controlled by strong pumped electric field ${ }^{20}$

The above mentioned QDs are either self-assembled ones or fabricated by confining electrons in quantum wells, where bulk phonons in substrates play an important role. ${ }^{2,7,13}$ In recent years, a new type of QDs based on self-assembled nanowires has been fabricated. ${ }^{21-25}$ The nanowires are perpendicular to the substrates, making the bulk phonons in the substrates less important than the quasi-one-dimensional confined phonons in the nanowires. 26,27 It has been shown that the confined phonons can lead to novel properties in optical absorption in nanowire-based single QDs and transport through nanowire-based DQDs ${ }^{28-30}$ Specifically, the spin relaxation in nanowire-based single QDs can be efficiently manipulated by external magnetic field due to the large phonon density of states (DOS) near the van Hove singularity of the confined phonons. ${ }^{31}$ However, the manipulation of spin relaxation by electric field in these nanowirebased QDs is not reported yet.

In this paper, we propose a scheme of manipulation of the spin relaxation in nanowire-based elongate DQDs by external electric field. In our system, the electric field can efficiently tune the energy spectrum of the DQDs where an anti-crossing between electron states with opposite majority spin exists. As the energy splittings between these states match certain van Hove singularity of the confined phonons, multiple sharp peaks occur in the electric field dependence of the spin relaxation rate (SRR). This feature offers an efficient scheme for the manipulation of the spin relaxation and hence on-and-off switches. Comparing to the electric spin relaxation manipulation in quantum-well-based DQDs, 17 the SRR in our system is much more sensitive to the electric field and can be manipulated with more flexibility. Moreover, due to the strong spin mixing in the vicinity of the anticrossing point, the off-diagonal density matrix elements have a large contribution to the SRR. Such contribution can have a pronounced impact on the SRR, which can be seen in the temperature dependence of the SRR. It should be noted that the strong spin mixing makes it difficult to define the majority spin for each state in the vicinity of the anti-crossing point. This makes the widely-used Fermi-golden-rule approach ${ }^{5,6,8-14}$ for spin relaxation calculation become obscure in this regime. So 
in the calculation, we apply the equation-of-motion approach developed by Jiang et al. $\stackrel{13}{1}^{1}$

We organize the paper as follows. In Sec. II, we introduce the model for our system and specify the Hamiltonian. The equation-of-motion approach is also briefly described in this section. In Sec. III, we first present the electric field dependence of the energy levels and the corresponding spin states of the DQDs. Then we show the electric field dependence of the SRR and demonstrate the electric manipulation of spin relaxation. Finally, we discuss the impact of the off-diagonal elements of the density matrix on the temperature dependence of the SRR. The validation of the Fermi-golden-rule is also addressed in this section. We summarize in Sec. IV.

\section{MODEL AND FORMALISM}

We consider a single-electron elongate DQDs embedded in InAs cylindrical nanowire with radius $R$. An external magnetic field $\boldsymbol{B}$ is applied along the wire (the $z$-axis). The total Hamiltonian can be expressed as

$$
H_{\text {tot }}=H_{\mathrm{e}}+H_{\mathrm{ph}}+H_{\mathrm{ep}},
$$

where $H_{\mathrm{e}}, H_{\mathrm{ph}}$ and $H_{\mathrm{ep}}$ are the electron, phonon and electron-phonon interaction Hamiltonian, respectively. The electron Hamiltonian has the form $H_{e}=H_{0}+H_{\mathrm{so}}$. Here $H_{0}$ is electron Hamiltonian without the SOC:

$$
H_{0}=\frac{\mathbf{p}^{2}}{2 m^{*}}+V_{c}(r)+V_{z}(z)+H_{\mathrm{B}},
$$

with $m^{*}$ denoting the electron effective mass. $V_{\mathrm{c}}(r)=$ $\frac{1}{2} m^{*} \omega_{0}^{2} r^{2}$ is the QD confinement in radial direction. Thus the dot has an effective diameter $d_{0}=\sqrt{\hbar \pi / m^{*} \omega_{0}}$. The confinement $V_{z}(z)$ in the $z$-axis is given by

$$
V_{z}(z)= \begin{cases}e E z+\frac{1}{2} e(a+2 d) E, & \frac{1}{2} a<|z|<\frac{1}{2} a+d \\ e E z+\frac{1}{2} e(a+2 d) E+V_{0}, & |z| \leq \frac{1}{2} a \\ \infty, & \text { otherwise }\end{cases}
$$

with $V_{0}$ representing the barrier height between the two dots, $a$ standing for the interdot distance and $d$ denoting the dot length. A schematic of $V_{z}(z)$ is plotted in Fig. 1(b)(IV). In our model, the radial confinement is much stronger than the longitudinal one, so we consider only the lowest electron subband in the radial direction. $H_{B}=\frac{1}{2} g \mu_{B} \boldsymbol{B} \cdot \boldsymbol{\sigma}$ is the Zeeman term with $g, \mu_{B}$ and $\sigma$ representing the electron g-factor, Bohr magneton and Pauli matrix, respectively. The SOC Hamiltonian takes the form

$$
H_{\mathrm{so}}=\frac{\gamma}{\hbar} \sigma_{y} p_{z}
$$

where $\gamma$ is the Rashba strength ${ }^{32,33}$

The confined phonon modes are calculated within isotropic elastic continuum model by assuming free-

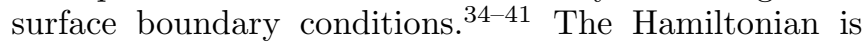

given by $H_{\mathrm{ph}}=\sum_{q \lambda} \hbar \omega_{q \lambda} a_{q \lambda}^{\dagger} a_{q \lambda}$, where $\omega_{q \lambda}$ is the eigenfrequency of the $\lambda$ th phonon mode with axial wave vector $q$, which is solely determined by the longitudinal or transverse sound velocity $v_{L}$ or $v_{T}$, respectively. Note that the calculated confined phonon modes can be classified into axial and radial modes according to the corresponding displacement field at small $q: \underline{30,42}$

Both the deformation potential and piezoelectric couplings are considered in our calculation. Assuming the displacement field of the confined phonons is $\boldsymbol{u}(\boldsymbol{r})$, the deformation potential coupling is given by the divergence of $\boldsymbol{u}(\boldsymbol{r}): H_{\mathrm{ep}}^{\mathrm{D}}(\boldsymbol{r})=-\Xi \boldsymbol{\nabla} \cdot \boldsymbol{u}(\boldsymbol{r})$, where $\Xi$ is the deformation coupling strength. The piezoelectric coupling is determined via $H_{\mathrm{ep}}^{\mathrm{P}}(\boldsymbol{r})=\frac{e}{\kappa} \int d \boldsymbol{r}_{e} \frac{\boldsymbol{\nabla} \cdot \boldsymbol{P}^{\mathrm{pz}}}{\boldsymbol{r}-\boldsymbol{r}_{e} \mid}$, where $\kappa$ denotes the relative dielectric constant, $e$ is the electron charge and $\boldsymbol{P}^{\mathrm{pz}}$ stands for the polarization induced by $\boldsymbol{u}(\boldsymbol{r}) \stackrel{35}{=}$ For InAs nanowires with wurtzite structure, $\boldsymbol{P}^{\mathrm{pz}}(\boldsymbol{r})=e_{15}\left[\partial_{z} u_{r}(\boldsymbol{r})+\partial_{r} u_{z}(\boldsymbol{r})\right] \boldsymbol{e}_{r}+\left[e_{31}\left(\partial_{r}+\frac{1}{r}\right) u_{r}(\boldsymbol{r})+\right.$ $\left.e_{33} \partial_{z} u_{z}(\boldsymbol{r})\right] \boldsymbol{e}_{z}$, where $e_{15}, e_{31}$ and $e_{33}$ are the piezoelectric constants $\stackrel{42.43}{ }$ By substituting $\boldsymbol{u}(\boldsymbol{r})$ into the expression of $H_{\mathrm{ep}}^{\mathrm{D}}$ and $H_{\mathrm{ep}}^{\mathrm{P}} \stackrel{42}{=}$ one can express the Hamiltonian of the electron-phonon interaction in the form

$$
H_{\mathrm{ep}}=\sum_{\eta=D, P} \sum_{q \lambda} M_{q \lambda}^{\eta} \chi_{q \lambda}^{\eta}\left(a_{q \lambda}+a_{-q \lambda}^{\dagger}\right),
$$

where $\left|M_{q \lambda}^{\mathrm{D}}\right|^{2}=\hbar \Xi^{2} /\left(2 \pi \rho_{m} \omega_{q \lambda} R^{2}\right)$ for the deformation potential coupling and $\left|M_{q \lambda}^{\mathrm{P}}\right|^{2}=8 \hbar \pi e^{2} e_{14}^{2} /\left(\kappa^{2} R^{2} \omega_{q \lambda}\right)$ for the piezoelectric coupling, with quantity $\rho_{m}$ being the mass density. The factor $\chi_{q \lambda}^{\eta}(\boldsymbol{r})$ depends on the confined phonon eigenmode. Note that since in the calculation, we consider only the lowest radial subband of the electron, only the dilatation phonon modes couple to the electron 30,44

Given the electron Hamiltonian $H_{e}$, the eigenstates $\{|\ell\rangle\}$ and corresponding eigenenergies $\left\{\varepsilon_{\ell}\right\}$ can be obtained by exact-diagonalization method $\stackrel{\underline{8}}{\text { The spin of }}$ each state can be calculated as $\langle\ell|\boldsymbol{S}| \ell\rangle$ with $\boldsymbol{S}=\frac{1}{2} \boldsymbol{\sigma}$. We apply the equation-of-motion approach to calculate the spin relaxation time. Following Ref. 13, we use the density matrix $\rho=\rho^{e} \otimes \rho^{\mathrm{ph}}$ to describe the system. Its time evolution in the interaction picture reads

$$
\begin{aligned}
\partial_{t} \rho^{\mathrm{I}}(t)= & -\frac{i}{\hbar}\left[V^{\mathrm{I}}(t), \rho(0)\right] \\
& -\frac{1}{\hbar^{2}} \int_{0}^{t} d \tau\left[V^{\mathrm{I}}(t),\left[V^{\mathrm{I}}(\tau), \rho^{\mathrm{I}}(\tau)\right]\right] .
\end{aligned}
$$

By applying the Born-Markovian approximation and carrying out the integral over the constant energy surface of the confined phonons, the equation-of-motion of the density matrix $\rho^{e}=\operatorname{tr}_{\mathrm{ph}}(\rho)$ for electron can be obtained from the above equation within the basis of the electron eigenstates (in Schrödinger picture) 13

$$
\begin{aligned}
\partial_{t} \rho_{m n}^{\mathrm{e}}= & -i \frac{\varepsilon_{m}-\varepsilon_{n}}{\hbar} \rho_{m n}^{\mathrm{e}}-\frac{1}{2} \sum_{\eta=D, P} \sum_{k l}\left\{W_{m k k l}^{\eta}\left(\varepsilon_{l k}\right) \rho_{l n}^{\mathrm{e}}\right. \\
& \left.-W_{m k l n}^{\eta}\left(\varepsilon_{l n}\right) \rho_{k l}^{\mathrm{e}}+\text { H.c. }\right\}
\end{aligned}
$$


with

$$
\begin{aligned}
W_{m k l n}^{\eta}\left(\varepsilon_{l n}\right)= & \sum_{q \lambda} \frac{\left|M_{q \lambda}^{\eta}\right|^{2} \chi_{q \lambda}^{\eta m k} \chi_{-q \lambda}^{\eta l n}}{\left|\partial_{q} \omega_{q \lambda}\right|} \\
& \times\left\{\left.\left[\bar{n}\left(\omega_{q \lambda}\right)+1\right] \theta\left(\varepsilon_{n l}\right)\right|_{\hbar \omega_{q \lambda}=\varepsilon_{n l}}\right. \\
& \left.+\left.\bar{n}\left(\omega_{q \lambda}\right) \theta\left(\varepsilon_{l n}\right)\right|_{\hbar \omega_{q \lambda}=\varepsilon_{l n}}\right\},
\end{aligned}
$$

in which $\chi_{q \lambda}^{\eta m n}=\left\langle m\left|\chi_{q \lambda}^{\eta}\right| n\right\rangle$ and $\theta\left(\varepsilon_{l n}\right)$ denotes the step function with $\varepsilon_{l n}=\varepsilon_{l}-\varepsilon_{n}$. Note that the transition rate $\Gamma_{\ell_{1} \ell_{2}}^{\eta}$ from state $\left|\ell_{1}\right\rangle$ to $\left|\ell_{2}\right\rangle$ can be expressed as $\Gamma_{\ell_{1} \ell_{2}}^{\eta}=$ $W_{\ell_{2} \ell_{1} \ell_{1} \ell_{2}}^{\eta}\left(\varepsilon_{\ell_{2} \ell_{1}}\right)$.

By applying a proper initial state, one can use the equation-of-motion of the density matrix to calculate the time evolution of $\left\langle S_{z}\right\rangle=\operatorname{tr}\left[\hat{\mathrm{S}}_{z} \rho^{\mathrm{e}}(\mathrm{t})\right]$, which is the expectation value of the $z$-component of the electron spin. We assume that the initial state is prepared by electrical spin injection ${ }^{45,46}$ or optical pumping of the electron spin,,$\underline{47}-\underline{49}$ which can polarize the electron spin along the external magnetic field. Thus density matrix elements in the basis of $\{|\ell\rangle\}$ for the initial state can be expressed as $\rho_{\ell \ell^{\prime}}^{e}(0)=\sum_{\xi}\langle\ell \mid \xi\rangle F_{\xi}\left\langle\xi \mid \ell^{\prime}\right\rangle$, where $\{|\xi\rangle\}$ are the spin-down eigenstates $\left(\left\langle\xi\left|S_{z}\right| \xi\right\rangle=-1 / 2\right)$ of $H_{0}$ with eigenenergies $\left\{\varepsilon_{\xi}\right\} . F_{\xi}=C \exp \left[-\varepsilon_{\xi} /\left(k_{B} T\right)\right]$ is the Maxwell-Boltzmann distribution with $C$ denoting the normalization parameter. Note that due to the SOC, $\rho^{e}(0)$ can have offdiagonal elements.

Given the time evolution of $\left\langle S_{z}\right\rangle$, we define the effective SRR as

$$
\bar{\tau}_{\alpha}^{-1}=(\ln \alpha) / \tau_{\alpha}
$$

with $\tau_{\alpha}$ being the time that the envelope of $\left\langle S_{z}\right\rangle$ decays to the $1 / \alpha$ from its initial value to its equilibrium value. Note that comparing to the SRR used in previous works,$\stackrel{13.50}{\longrightarrow}$ we introduce an additional factor $\ln \alpha$ in order to guarantee that if $\left\langle S_{z}\right\rangle$ can be well-described by a single exponential decay, $\bar{\tau}_{\alpha}^{-1}$ is independent on $\alpha$. This is helpful when we compare the effective SRR $\bar{\tau}_{\alpha}^{-1}$ with different $\alpha$. It should be emphasized that if $\left\langle S_{z}\right\rangle$ does not follow a single exponential decay, the effective SRR $\bar{\tau}_{\alpha}^{-1}$ is $\alpha$-dependent. One should properly choose $\alpha$ to give an overall description of the spin relaxation in the regime of investigation.

\section{NUMERICAL RESULTS}

In the computation, we consider a DQD with length $d=50 \mathrm{~nm}$ for each dot, interdot distance $a=10 \mathrm{~nm}$, barrier height $V_{0}=0.3 \mathrm{eV}$ and effective dot diameter $d_{0}=12 \mathrm{~nm}$. The Rashba strength $\gamma$ is set to be $2.4 \times 10^{-11} \mathrm{eV} \cdot \mathrm{m}$, corresponding to the spin-orbit length $\lambda_{\text {so }}=\hbar^{2} /\left(m^{*} \gamma\right)=133 \mathrm{~nm}$ which is close to the value $\lambda_{\text {so }} \sim 127 \mathrm{~nm}$ reported in Ref. 25. The radius of nanowire $R$ is set to be $30 \mathrm{~nm}$. The InAs nanowires have wurtzite crystal structure with orientation along the
[0001] direction ${ }^{25,51}$ The piezoelectric constants for the wurtzite InAs can be expressed as $e_{15}=e_{31}=-e_{33} / 2=$ $-e_{14} / \sqrt{3}$ with $e_{14}$ being the piezoelectric constant for bulk InAs. $\frac{42}{=}$ Note that although both the deformation potential and piezoelectric couplings are considered in our computation, we find that the piezoelectric coupling is dominant in our system. The other parameters used in our calculation are listed in Table I.

TABLE I. Material parameters used in the computation (from Ref. $\underline{52}$ unless otherwise specified).

\begin{tabular}{llll}
\hline \hline$\rho_{m}$ & $5900 \mathrm{~kg} / \mathrm{m}^{3}$ & $\Xi$ & $5.8 \mathrm{eV}$ \\
$v_{T}$ & $2130 \mathrm{~m} / \mathrm{s}$ & $m^{*}$ & $0.0239 m_{0}$ \\
$v_{L}$ & $4410 \mathrm{~m} / \mathrm{s}$ & $\kappa$ & 15.15 \\
$e_{14}$ & $3.5 \times 10^{8} \mathrm{~V} / \mathrm{m}$ & $g$ & $-9.0^{\mathrm{a}}$ \\
\hline \hline
\end{tabular}

${ }^{\text {a }}$ Ref. 25.

\section{A. Energy levels and spin configurations}

Before we study the behavior of the effective SRR, it is helpful to first discuss the electric field dependence of energies and spins of electron states in the DQD. We plot the lowest three energy levels of the DQD as function of electric field in Fig. 1(a) with external magnetic field $B=1 \mathrm{~T}$. An anti-crossing between the first excited state $|2\rangle$ and the second excited state $|3\rangle$ can be clearly identified in the energy levels which is induced by the coaction of the electric field, the magnetic field and the SOC. The position of the anti-crossing point depends on the magnetic field, which is shown in Fig. 1(a) by dots at some selected magnetic fields. The arrows in Fig. 1(a) illustrate the spin of each state along the $z$-axis defined as $\left\langle\ell\left|S_{z}\right| \ell\right\rangle$ (The other spin components are zero). With the increase of the electric field, the spins of the lowest three levels $(|1\rangle,|2\rangle$ and $|3\rangle)$ change from $\uparrow, \uparrow$ and $\downarrow$ (before the anti-crossing point) to $\uparrow, \downarrow$ and $\uparrow$ (after the anti-crossing point), respectively. In the vicinity of the anti-crossing point, due to the spin mixing induced by the SOC, the spins of the states $|2\rangle$ and $|3\rangle$ decrease and tend to zero at the anti-crossing point, while the spin of $|1\rangle$ is not sensitive to the electric field.

The electron spin states can be better illustrated by the spin configuration of each state, e.g., the distribution of each spin state as function of $z$. We plot the spin configurations of the three states in Fig. 1(b) at three typical electric fields $E_{\mathrm{I}} / E_{\mathrm{II}} / E_{\mathrm{III}}$ corresponding to the fields before/at/after the anti-crossing point. The component $\left\langle S_{y}\right\rangle$ of the spin configuration is always zero for all the three states so it is not plotted in the figure. One finds that the spin configuration of the ground state is not sensitive to the electric field and it is mainly distributed in the left dot for not too small electric field 

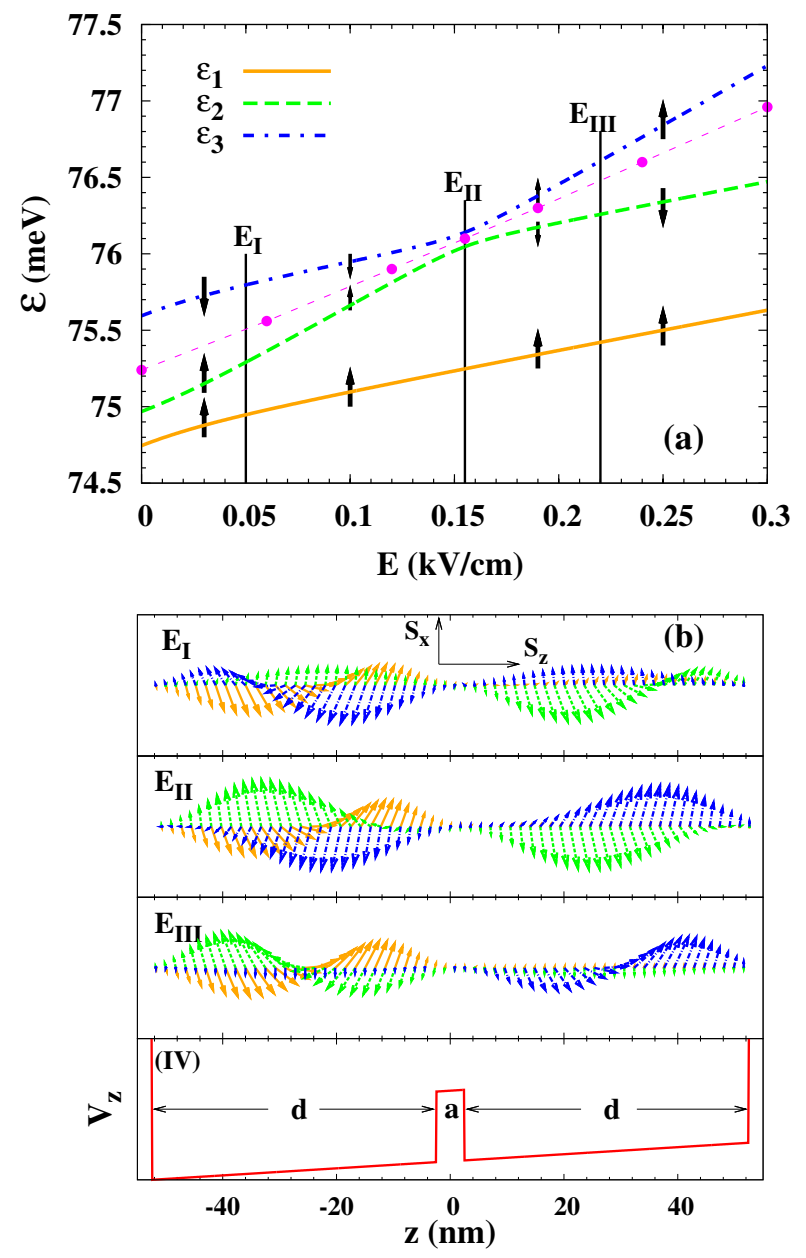

FIG. 1: (Color online) (a) The lowest three energy levels vs the electric field $E$ with the magnetic field $B=1 \mathrm{~T}$. The arrows indicate the spins of the states. Longer arrow means larger spin. The thin pink dashed curve with dots represents the position of the anti-crossing point between the states $|2\rangle$ and $|3\rangle$ at different magnetic fields, where the corresponding magnetic fields of the dots are $0.27 \mathrm{~T}, 0.46 \mathrm{~T}, 0.81 \mathrm{~T}, 1.0 \mathrm{~T}$, $1.16 \mathrm{~T}, 1.53 \mathrm{~T}$ and $1.9 \mathrm{~T}$, respectively (from left to right). The three vertical lines label the typical electric fields $E_{\mathrm{I}}=$ $0.05 \mathrm{kV} / \mathrm{cm}, E_{\mathrm{II}}=0.156 \mathrm{kV} / \mathrm{cm}$ and $E_{\mathrm{III}}=0.22 \mathrm{kV} / \mathrm{cm}$ corresponding to the regime before, at and after the anticrossing point. (b) The spin configurations of the lowest three energy levels as function of $z$ for the three typical electric fields $E_{\mathrm{I}}, E_{\mathrm{II}}$ and $E_{\mathrm{III}}$. Arrow in (b) and curve in (a) with the same color and line type stand for the same state. The schematic of the potential of the DQD is shown in (b)(IV).

$(E>0.01 \mathrm{kV} / \mathrm{cm})$. The effect of the electric field on the spin configuration is pronounced for the two excited states. There are two types of spin configurations for the excited states far away from the anti-crossing point: (i) the spin configuration distributes mainly in the right dot and has a similar structure as the spin configuration of the ground state in the left dot; (ii) the spin configuration distributes mainly in the left dot and has a different structure as the ground state. Before the anti-crossing point [Fig. [1(b) $\left.\left(\mathrm{E}_{\mathrm{I}}\right)\right],|2\rangle /|3\rangle$ belongs to type (i)/(ii). In contrast, after the anti-crossing point [Fig. [1(b)( $\left.\mathrm{E}_{\mathrm{III}}\right)$ ], $|2\rangle /|3\rangle$ belongs to type (ii)/(i). In the vicinity of the anticrossing point [Fig. [1(b) $\left.\left(\mathrm{E}_{\mathrm{II}}\right)\right]$, both excited states belong to a new type of spin configuration, which is evenly distributed in the DQD and polarized mainly along the $x$ axis. This suggests that there is a strong spin mixing between the two excited states, which makes the expectation value of the electron spin $\left\langle S_{z}\right\rangle$ associate with not only the diagonal elements but also the off-diagonal elements between the two states in the density matrix.

As both the energies and spins of the two excited states can be efficiently manipulated in the vicinity of the anticrossing point, one would expect that they should have a pronounced impact on the spin relaxation. So in the following discussion, we will concentrate on the lowest three levels.

\section{B. Effective spin relaxation rate}

With the equation-of-motion approach we applied here, the effective SRR is obtained from the time evolution of $\left\langle S_{z}\right\rangle$, which has different behaviors under different electric/magnetic fields and temperatures. So we start our discussion of the spin relaxation by studying the typical time evolution of $\left\langle S_{z}\right\rangle$. Then we study the electric dependence of the effective SRR and demonstrate how the electric spin relaxation manipulation can be achieved in our system. We also compare our scheme to the scheme based on DQDs in quantum wells. Finally, by studying the temperature dependence, we also show the importance of the off-diagonal elements of the density matrix to the spin relaxation.

\section{Typical behavior of $\left\langle S_{z}\right\rangle$}

We show the typical behaviors of time evolution of $\left\langle S_{z}\right\rangle$ in both the short- and long-time regimes at different electric fields in Fig. 2(a) (d). Generally speaking, they can be classified into two types: (i) single-exponential decay [Fig. 2(a)] and (ii) double-exponential decay [Fig. 2(b), (c) and (d)]. For type (i), the time evolution of $\left\langle S_{z}\right\rangle$ [dots in Fig. 2(a)] can be well-fitted by a single-exponential function $f_{1}(t)=a_{1} e^{-t / \tau_{\mathrm{E}_{1}}}+b_{1}$ [blue dashed curve in Fig. 2(a)] with $a_{1}, b_{1}$ and $\tau_{\mathrm{E}_{1}}$ being fitting parameters. For type (ii), the time evolution of $\left\langle S_{z}\right\rangle$ [dots in Fig. 2(b), (c) and (d)] has two stages: a short-time stage where $\left\langle S_{z}\right\rangle$ decays faster and a long-time stage where $\left\langle S_{z}\right\rangle$ decays slower. The relative importance of the two stages can be different. For example, the short-time stage is important in Fig. 2(b) since it leads to a large variation of $\left\langle S_{z}\right\rangle$ from -0.5 to 0.3 . While in Fig. 2(d), the long-time stage is important. Note that usually the time evolution of $\left\langle S_{z}\right\rangle$ in the short-time stage is much faster than that in the long-time stage. It is also worth noting that a strong 

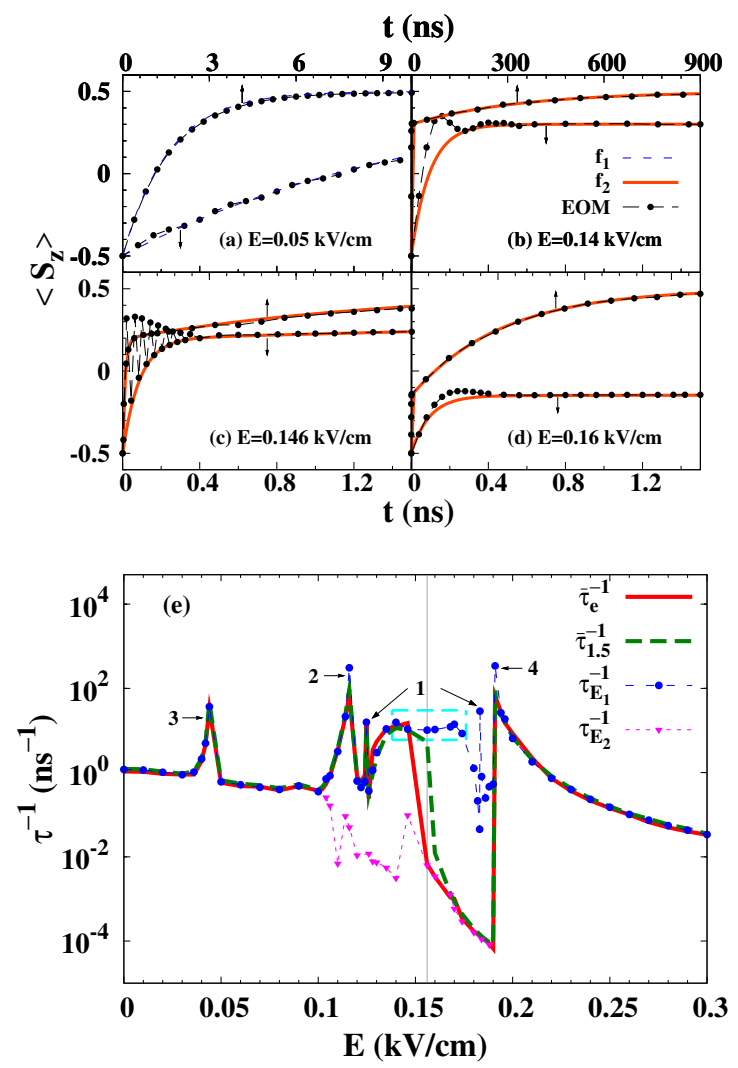

FIG. 2: (Color online) (a) $\sim$ (d) Time evolution of $\left\langle S_{z}\right\rangle$ in both the short- (lower scale) and long-time (upper scale) regimes with $B=1 \mathrm{~T}$ and $T=0 \mathrm{~K}$ at the electric field $E=0.05,0.14,0.146$ and $0.16 \mathrm{kV} / \mathrm{cm}$, respectively. Black dashed curve with dots: $\left\langle S_{z}\right\rangle$ from the equation-of-motion approach. Blue dashed curve: best-fitting with single exponential decay. Red solid curve: best-fitting with double exponential decay. (e) SRRs as function of electric field with $B=1 \mathrm{~T}$ and $T=0 \mathrm{~K}$. Red solid/Green dashed curve: effective SRR $\bar{\tau}_{\mathrm{e}}^{-1} / \bar{\tau}_{1.5}^{-1}$. Blue dots/Pink triangles: rate for the first/second spin relaxation process. The cyan dashed box indicates the vicinity of the anti-crossing point. The gray vertical line indicates the position of the anti-crossing point.

oscillation can exist in the short-time stage, as shown in Fig. 2(c). The evolution of $\left\langle S_{z}\right\rangle$ can be fitted by a double exponential function $f_{2}(t)=a_{1} e^{-t / \tau_{\mathrm{E}_{1}}}+a_{2} e^{-t / \tau_{\mathrm{E}_{2}}}+b$ [orange-red solid curves in Fig. 2(b) (d)] with $a_{1}, a_{2}, b$, $\tau_{\mathrm{E}_{1}}$ and $\tau_{\mathrm{E}_{2}}$ being fitting parameters.

The behavior of $\left\langle S_{z}\right\rangle$ suggests that there are two main spin relaxation processes in the system: the first process with the characteristic rate $\tau_{\mathrm{E}_{1}}^{-1}$, which dominates in the short-time stage and the second process with the characteristic rate $\tau_{\mathrm{E}_{2}}^{-1}$, which dominates in the long-time stage. If the time evolution of $\left\langle S_{z}\right\rangle$ belongs to type (i), only one process dominates (corresponding to $\tau_{\mathrm{E}_{1}}^{-1}$ ), which solely determines the effective SRRs. If the time evolution of $\left\langle S_{z}\right\rangle$ belongs to type (ii), both processes can contribute to the effective SRRs. The effective SRR $\bar{\tau}_{\alpha}^{-1}$ is given by the average over the two rates $\tau_{\mathrm{E}_{1}}^{-1}$ and $\tau_{\mathrm{E}_{2}}^{-1}$ with the weight determined by the parameter $\alpha$. For small $\alpha$, the effective SRR $\bar{\tau}_{\alpha}^{-1}$ mainly describes the short time behavior and hence has a large weight of $\tau_{\mathrm{E}_{1}}^{-1}$. While for large $\alpha, \tau_{\mathrm{E}_{2}}^{-1}$ makes a large contribution to the $\bar{\tau}_{\alpha}^{-1}$.

To give a comprehensive description of both the shortand long-time behaviors of the spin relaxation, we choose the effective SRR $\bar{\tau}_{\alpha}^{-1}$ with two typical values of $\alpha: \alpha=$ 1.5 which better describes the short-time behavior and $\alpha=e$ which better describes the long-time behavior.

\section{Electric field dependence}

The effective SRRs $\bar{\tau}_{1.5}^{-1}$ and $\bar{\tau}_{\mathrm{e}}^{-1}$ as function of electric field at magnetic field $B=1 \mathrm{~T}$ and temperature $T=0 \mathrm{~K}$ are plotted in Fig. 2(e). One can see that both effective SRRs have similar electric field dependence with two main features: one is the large drop at the anti-crossing point, the other is the multiple sharp peaks indicated by the numbered arrows.

To understand these features, it is helpful to first identify the dominant spin relaxation process at different electric fields. This can be done by comparing the effective SRRs $\bar{\tau}_{1.5}^{-1}$ and $\bar{\tau}_{\mathrm{e}}^{-1}$ to the two rates $\tau_{\mathrm{E}_{1}}^{-1}$ and $\tau_{\mathrm{E}_{2}}^{-1}$ in Fig. 2(e). Far from the anti-crossing point $(E<0.102 \mathrm{kV} / \mathrm{cm}$ and $E>0.19 \mathrm{kV} / \mathrm{cm})$, only one spin relaxation process dominates. Both effective SRRs are determined by the corresponding rate $\tau_{\mathrm{E}_{1}}^{-1}$. For the electric field $E$ in the vicinity of the anti-crossing point $[E \in(0.102,0.19) \mathrm{kV} / \mathrm{cm}]$, the second spin relaxation process becomes important and can dominate the effective SRRs after the anti-crossing point. The difference of the two effective SRRs $\bar{\tau}_{1.5}^{-1}$ and $\bar{\tau}_{\mathrm{e}}^{-1}$ at the anti-crossing point is due to the fact that the rate $\tau_{\mathrm{E}_{1}}^{-1}$ has a smaller contribution to $\bar{\tau}_{\mathrm{e}}^{-1}$ than to $\bar{\tau}_{1.5}^{-1}$.

Having identified the dominant spin relaxation process, one finds that the first feature, e.g., the large drop at the anti-crossing point, is due to the relative importance of the two spin relaxation processes: the second spin relaxation process becomes more and more important as the electric field $E$ passes the anti-crossing point. The second feature, e.g., the multiple sharp peaks, is solely induced by the spin relaxation process corresponding to $\tau_{\mathrm{E}_{1}}^{-1}$.

The relative importance of the two processes can be understood by studying the relevant energy levels. This can be done by comparing the transition rates between the lowest three energy levels to the rates $\tau_{\mathrm{E}_{1}}^{-1}$ and $\tau_{\mathrm{E}_{2}}^{-1}$ in Fig. 3(a). The first spin relaxation process corresponding to $\tau_{\mathrm{E}_{1}}^{-1}$ is determined by different transitions at different electric fields. For $E<0.19 \mathrm{kV} / \mathrm{cm}$, this process is mainly determined by the transition $|3\rangle \rightarrow|1\rangle$ or $|3\rangle \rightarrow|2\rangle$. The transition with larger transition rate dominates this process. For $E>0.19 \mathrm{kV} / \mathrm{cm}$, this process is determined by the transition $|2\rangle \rightarrow|1\rangle$. The second spin relaxation process corresponding to $\tau_{\mathrm{E}_{2}}^{-1}$, which only exists in the regime $E \in(0.102,0.19) \mathrm{kV} / \mathrm{cm}$, is determined by the transition $|2\rangle \rightarrow|1\rangle$. So the dominant spin relax- 

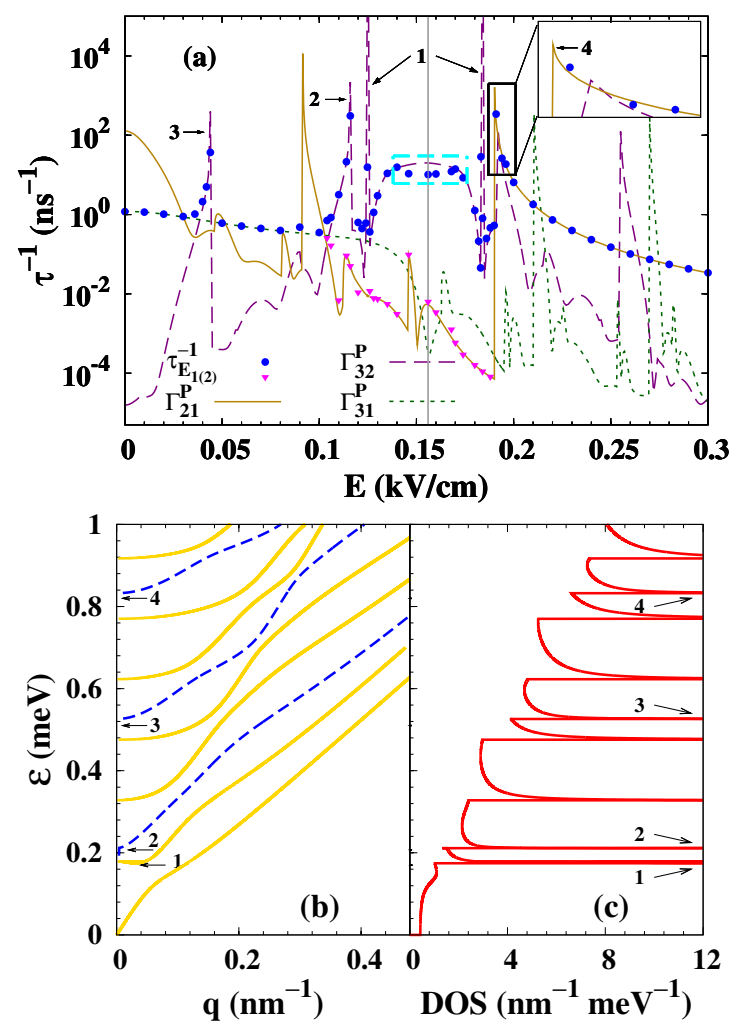

FIG. 3: (Color online) (a) Transition rates $\Gamma_{21}^{\mathrm{P}}$ (yellow solid curve), $\Gamma_{32}^{\mathrm{P}}$ (purple dashed curve) and $\Gamma_{31}^{\mathrm{P}}$ (green dotted curve) due to the piezoelectric coupling only, with $B=$ $1 \mathrm{~T}$ and $T=0 \mathrm{~K}$. Blue dots/Pink triangles: rate for the first/second spin relaxation process. Inset: the enlargement of the peak 4 . The cyan dashed box indicates the vicinity of the anti-crossing point and the gray vertical line indicates the position of the anti-crossing point, which are the same with those in Fig. 2(b). (b) Spectrum of the confined phonons in the nanowire. The orange solid/blue dashed curves represent the axial/radial phonon modes. (c) DOS of the confined phonons. The arrows with numbers in (b) and (c) indicate the van Hove singularities which are responsible for the sharp peaks labelled with the same numbers in (a).

ation process changes from $|3\rangle \rightarrow|2\rangle$ to $|2\rangle \rightarrow|1\rangle$ as the electric field $E$ passes the anti-crossing point, which agrees with the spins of the states shown in Fig. 1(a). This explains the large drop at the anti-crossing point. Note that in the calculation of the transition rate, we consider only the piezoelectric coupling since it is dominant in our system 30

Now let us turn to the second feature of the effective SRRs, e.g., the multiple sharp peaks, which are determined by the first spin relaxation process corresponding to $\tau_{\mathrm{E}_{1}}^{-1}$. These sharp peaks occur as the energy splitting between the two states of the spin relaxation process matches the van Hove singularities of the confined phonons, e.g., the denominator of the transition rate $\Gamma_{\ell_{1} \ell_{2}}^{\mathrm{P}}$ tends to zero $\stackrel{31}{=}$ According to Fig. 3(a), one can see that the peaks 2,3 and the double peaks 1 are induced by the transition $|3\rangle \rightarrow|2\rangle$ whereas the peak 4 is induced by the transition $|2\rangle \rightarrow|1\rangle$. By comparing the energy levels and corresponding spin configurations of the DQD shown in Fig. 1(a) to the spectrum and DOS of the confined phonons in Fig. 3(b) and (c), one can also identify the corresponding confined phonon mode and van Hove singularity for each peak, which are indicated by the arrows with the same numbers in Fig. B(b) and (c), respectively.

It should be emphasized that not every van Hove singularity can induce a sharp peak. This is because the behavior of the nominator of the coefficient $\Gamma_{\ell_{1} \ell_{2}}^{\mathrm{P}}$, e.g., the form factor, is also crucial for the peaks, which depends on the properties of the confined phonon modes and the electron-phonon interactions. Zeros of the form factors can greatly suppress the SRR. To induce a sharp peak, the suppression must be absent or weak at the corresponding van Hove singularity. Two types of van Hove singularities satisfy such condition in our system. The first type is the van Hove singularities with $q=0$ for the radial phonon modes. The peaks (peaks 2, 3 and 4 ) exist since the corresponding form factor of the piezoelectric coupling tends to zero slow enough so that the suppression effect is weak. The second type is the van Hove singularities with phonon wave vector $q \neq 0$. The corresponding peaks (the double peaks 1 ) are divergent due to the absence of zeros of the form factors at $q \neq 0 \underline{\underline{31}}$ Both the deformation potential and piezoelectric couplings can induce such peaks.

These peaks can be used for electric spin relaxation manipulation. For example, around peak 3, by changing the electric field within $0.01 \mathrm{kV} / \mathrm{cm}$, the effective SRR can change up to 2 orders of magnitude. While for the electric field a little far away from peak 3, the effective SRRs become rather insensitive to the electric field. Thus it can be used as electric on-and-off spin switches. It is worth comparing our spin relaxation manipulation scheme to the one based on GaAs DQDs in quantum wells where bulk phonons dominate discussed by Wang and $\mathrm{Wu}$ in Ref. 17. There, the spin relaxation manipulation relies on the fact that, for the vertical DQDs considered in their scheme, the electron-phonon scattering between the bonding and anti-bonding orbital states of the DQDs has a very large contribution to the SRR for small electric field. The SRR can be greatly suppressed by breaking these states by strong electric field, resulting in the spin relaxation manipulation. As the bonding/anti-bonding state plays the central role in the quantum-well based DQD scheme, the high energy anti-bonding state must be occupied or serve as the virtual state for the hyperfine-mediated spin relaxation process. So their scheme works with high electric field (to break the bonding/anti-bonding states) at high temperature (to make the high energy anti-bonding state occupied) or weak magnetic field (to ensure the hyperfinemediated spin relaxation is not suppressed). In our scheme, the spin relaxation manipulation relies mainly 
on the quasi-one-dimensional properties of the confined phonons, which does not suffer from such restrictions. It can also work at low temperature, strong magnetic field or weak electric field. Moreover, the spin relaxation manipulation can be accomplished at different electric fields, which can be tuned by the nanowire radius $R$. Thus our scheme is more sensitive and flexible than the scheme in quantum-well-based DQDs.

In the above discussion, facilitated with the transition rates, we have explained the two main features of the effective SRR from the equation-of-motion approach. It should be emphasized that in such explanation based on the transition rate, only the contribution of the diagonal elements of the density matrix to the spin relaxation is considered. This is valid when the electric field is far away from the anti-crossing point. However, for the electric field sufficiently close to the anti-crossing point, the contribution of the off-diagonal elements becomes important, which can have a pronounced effect on the spin relaxation. The spin relaxation in this case cannot be explained by the transition rate. This can be seen in the cyan dashed box in Fig. 3(a) where $\Gamma_{32}^{\mathrm{P}}$ overestimates the rate $\tau_{\mathrm{E}_{1}}^{-1}$ induced by the transition $|3\rangle \rightarrow|2\rangle$.

In fact, the rate $\tau_{\mathrm{E}_{1}}^{-1}$ at the anti-crossing point $E=$ $0.156 \mathrm{kV} / \mathrm{cm}$ is equal to $\Gamma_{32}^{\mathrm{P}} / 2$, which is just the relaxation rate of the off-diagonal elements between the states $|3\rangle$ and $|2\rangle$. This suggests that the off-diagonal elements dominate the spin relaxation at this point. This can be seen more clearly from the temperature dependence of the effective SRR in the next section.

\section{Temperature dependence}

Now we discuss the temperature dependence of the effective SRRs at the anti-crossing point with $E=$ $0.156 \mathrm{kV} / \mathrm{cm}$ and $B=1 \mathrm{~T}$, shown in Fig. 4(a). In the temperature regime we consider here, the contribution of the high energy levels can still be neglected, so only the lowest three energy levels are important. The most prominent feature of the effective SRRs is the existence of the smooth peak for $\bar{\tau}_{1.5}^{-1}$ at $T=7 \mathrm{~K}$, while $\bar{\tau}_{\mathrm{e}}^{-1}$ is monotonically increasing. Comparing to the two rates $\tau_{\mathrm{E}_{1}}^{-1}$ and $\tau_{\mathrm{E}_{2}}^{-1}$, one finds that $\bar{\tau}_{1.5}^{-1}$ is mainly determined by the spin relaxation process corresponding to $\tau_{\mathrm{E}_{1}}^{-1}$, which is due to the transition $|3\rangle \rightarrow|2\rangle$ at the anti-crossing point. While $\bar{\tau}_{\mathrm{E}_{\mathrm{e}}}^{-1}$ is mainly determined by $\tau_{\mathrm{E}_{2}}^{-1}$ which is induced by the transition $|2\rangle \rightarrow|1\rangle$. The peak is mainly due to the transition $|3\rangle \rightarrow|2\rangle$, so the peak in $\bar{\tau}_{1.5}^{-1}$ is absent in $\bar{\tau}_{\mathrm{e}}^{-1}$. Note that the behaviors of the time evolution of $\left\langle S_{z}\right\rangle$ from the equation-of-motion approach with the temperature before and after the peak are different, which can be seen in Fig. $4(\mathrm{~b}) \sim(\mathrm{e})$. Although both can be fitted by the double exponential function, $\left\langle S_{z}\right\rangle$ exhibits an oscillation in the first spin relaxation process before the peak [Fig. 4 (b)(c)] while the oscillation vanishes after the peak [Fig. $4(\mathrm{~d})(\mathrm{e})]$.
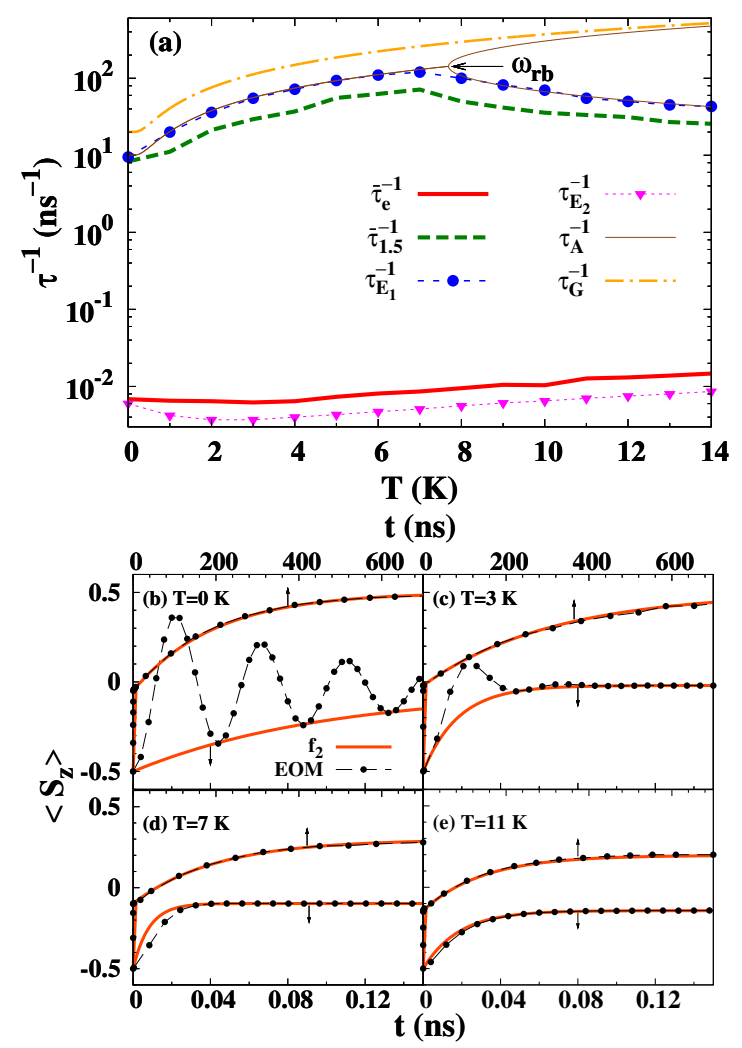

FIG. 4: (Color online) (a) SRRs as function of the temperature $T$ with $E=0.156 \mathrm{kV} / \mathrm{cm}$ and $B=1 \mathrm{~T}$. Curves (dots) with the same color and type represent the same quantities in Fig. 2(e). Brown solid thin curve: the relaxation rate from Eq. (10). Yellow dashed-dotted curve: the SRR from the Fermi-golden-rule approach. See text for details. (b) $\sim(\mathrm{e})$ Time evolution of $\left\langle S_{z}\right\rangle$ in both the short- (lower scale) and long-time (upper scale) regimes with $E=0.156 \mathrm{kV} / \mathrm{cm}$ and $B=1 \mathrm{~T}$ at temperature $T=0,3,7$ and $11 \mathrm{~K}$, respectively. Curves with the same color and type represent the same quantities in Fig. 2

The above-mentioned behavior can be understood if we assume the off-diagonal elements of the density matrix between the two states $|2\rangle$ and $|3\rangle$ dominate the spin relaxation. As far as the two states are concerned, the time evolution of $\left\langle S_{z}\right\rangle$ due to the off-diagonal elements follows an oscillation decay: $\left\langle S_{z}(t)\right\rangle=A \sin (\Omega t) e^{-t / T_{2}}+$ $B$, where $\Omega=\sqrt{\omega_{\mathrm{rb}}^{2}-T_{2}^{-2}}$ with $\omega_{\mathrm{rb}}$ representing the Rabi frequency and $T_{2}^{-1}=\Gamma_{32}^{\mathrm{P}} / 2$ being the relaxation rate. The parameters $A$ and $B$ are constants. As the temperature increases, the oscillation becomes slower due to the increase of the transition rate $\Gamma_{32}^{\mathrm{P}}$ and vanishes when $T_{2}^{-1}>\omega_{\mathrm{rb}}$, contributing another exponent decay to $\left\langle S_{z}\right\rangle$. This explains the vanishing of the oscillation in $\left\langle S_{z}\right\rangle$ from the equation-of-motion approach at high temperature.

According to the previous discussion, one can also give an analytical estimation for the relaxation rate induced 
by the off-diagonal elements of the density matrix between $|3\rangle$ and $|2\rangle$ :

$$
\tau_{\mathrm{A}}^{-1}=T_{2}^{-1} \pm \theta\left(T_{2}^{-1}-\omega_{\mathrm{rb}}\right) \tilde{\Omega},
$$

where $\tilde{\Omega}=\sqrt{T_{2}^{-2}-\omega_{\mathrm{rb}}^{2}}$. We plot $\tau_{\mathrm{A}}^{-1}$ in Fig. 幽(a), which agrees with $\tau_{\mathrm{E}_{1}}^{-1}$ very well. Note that the upper branch of $\tau_{\mathrm{A}}^{-1}$ [corresponding to "+" in Eq. (10)] cannot be obtained from the equation-of-motion approach since its contribution to the spin relaxation is very small thus is difficult to be fitted numerically.

It is noted that this peak is difficult to obtain for the quantum-well based QDs where bulk phonons dominate. This is mainly due to the fact that the transition rate is suppressed by the small DOS of the bulk phonons when the energy splitting between the two states becomes small, 54 making it difficult to fulfill the condition $T_{2}^{-1}>\omega_{\mathrm{rb}}$. Whereas the DOS of the confined phonons is almost constant away from the van Hove singularities, it is easy to have the condition $T_{2}^{-1}>\omega_{\mathrm{rb}}$ satisfied.

It is pointed out that the large contribution of the offdiagonal elements makes the widely-used Fermi-goldenrule approach,,$\frac{8.10-14}{14}$ which calculates the SRR by the formula $\tau_{\mathrm{G}}^{-1}=\sum_{\eta=\mathrm{D}, \mathrm{P}} \sum_{\ell_{1} \ell_{2}} F_{\ell_{1}} \Gamma_{\ell_{1} \ell_{2}}^{\eta}$, inapplicable. As an example, we plot the temperature dependence of the SRR $\tau_{\mathrm{G}}^{-1}$ from the Fermi-golden-rule approach in Fig. 4(a) with yellow dashed-dotted curve. It exhibits an monotonic increase without the temperature peak. Moreover, it overestimates the SRR in the whole temperature regime. This suggests that for the system with strong spin mixing, the Fermi-golden-rule approach is inadequate. To calculate the SRR properly, one has to apply the equation-of-motion approach.

\section{SUMMARY}

In summary, we have investigated the effective SRRs induced by confined phonons in the nanowire-based
DQDs. We first study the energies and spins of the lowest three states and find that both can be efficiently tuned by the electric field. An anti-crossing exists between the first and second excited states, which is due to the coaction of the electric field, the magnetic field and the SOC. Then we study the electric field dependence of the effective SRR within the equation-of-motion approach and find that multiple sharp peaks exist in the effective SRR, which can be used as the electric on-and-off spin switches. The confined phonons are found to be crucial for these peaks. The quasi-one-dimensional confined phonons exhibit extremely strong DOS at the van Hove singularities, resulting in the multiple sharp peaks as the energy splittings between states with different spins match certain van Hove singularities. Both the van Hove singularities with $q \neq 0$ and the ones with $q=0$ of radial confined phonon modes can induce such peaks, whilst the piezoelectric coupling is found to be crucial for the latter. The manipulation of the spin relaxation based on these multiple sharp peaks offers on-and-off spin switches, which is more sensitive and flexible than the one based on DQDs in quantum wells. We also find that the contribution of the off-diagonal elements of the density matrix can dominate the spin relaxation at the anti-crossing point due to the large spin mixing, resulting in a smooth peak in the temperature dependence of the effective SRR. This makes the widely-used Fermi-golden-rule approach inadequate in the vicinity of the anti-crossing point. These results show a great potential of the nanowire-based DQDs in the spin-based quantum information processing and spintronic devices.

\section{Acknowledgments}

This work was supported by the Natural Science Foundation of China under Grant No. 10725417. One of the authors (YY) was also partially supported by the China Postdoctoral Science Foundation.
* Author to whom correspondence should be addressed; Electronic address: mwwu@ustc.edu.cn.

1 J. M. Taylor, H.-A. Engel, W. Dur, A. Yacoby, C. M. Marcus, P. Zoller, and M. D. Lukin, Nat. Phys. 1, 177 (2005).

2 R. Hanson, L. P. Kouwenhoven, J. R. Petta, S. Tarucha, and L. M. K. Vandersypen, Rev. Mod. Phys. 79, 1217 (2007).

3 B. Trauzettel, M. Borhani, M. Trif, and D. Loss, J. Phys. Soc. Jpn. 77, 031012 (2008).

4 R. Hanson and D. D. Awschalom, Nature 453, 1043 (2008).

5 A. V. Khaetskii and Y. V. Nazarov, Phys. Rev. B 61, 12639 (2000); 64, 125316 (2001).

6 L. M. Woods, T. L. Reinecke, and Y. Lyanda-Geller, Phys. Rev. B 66, 161318(R) (2002).

7 V. N. Golovach, A. Khaetskii, and D. Loss, Phys. Rev.
Lett. 93, 016601 (2004).

8 J. L. Cheng, M. W. Wu, and C. Lü, Phys. Rev. B 69, 115318 (2004).

9 S. I. Erlingsson and Y. V. Nazarov, Phys. Rev. B 66, 155327 (2002).

10 V. A. Abalmassov and F. Marquardt, Phys. Rev. B 70, 075313 (2004).

11 P. Stano and J. Fabian, Phys. Rev. B 74, 045320 (2006); 77, 045310 (2008).

12 D. V. Bulaev and D. Loss, Phys. Rev. B 71, 205324 (2005).

13 J. H. Jiang, Y. Y. Wang, and M. W. Wu, Phys. Rev. B 77, 035323 (2008).

14 C. F. Destefani and S. E. Ulloa, Phys. Rev. B 72, 115326 (2005).

15 Y. Tokura, W. G. van der Wiel, T. Obata, and S. Tarucha, Phys. Rev. Lett. 96, 047202 (2006). 
16 J. R. Petta, A. C. Johnson, A. Yacoby, C. M. Marcus, M. P. Hanson, and A. C. Gossard, Phys. Rev. B 72, 161301(R) (2005).

17 Y. Y. Wang and M. W. Wu, Phys. Rev. B 74, 165312 (2006); 77, 125323 (2008).

18 K. C. Nowack, F. H. L. Koppens, Y. V. Nazarov, and L. M. K. Vandersypen, Science 318, 1430 (2007).

19 M. Pioro-Ladriere, T. Obata, Y. Tokura, Y.-S. Shin, T. Kubo, K. Yoshida, T. Taniyama, and S. Tarucha, Nat. Phys. 4, 776 (2008).

20 D. V. Khomitsky and E. Y. Sherman, Europhys. Lett. 90, 27010 (2010).

${ }^{21}$ M. T. Björk, B. J. Ohlsson, T. Sass, A. I. Persson, C. Thelander, M. H. Magnusson, K. Deppert, L. R. Wallenberg, and L. Samuelson, Appl. Phys. Lett. 80, 1058 (2002).

${ }^{22}$ M. T. Björk, C. Thelander, A. E. Hansen, L. E. Jensen, M. W. Larsson, L. R. Wallenberg, and L. Samuelson, Nano Lett. 4, 1621 (2004).

23 M. T. Björk, A. Fuhrer, A. E. Hansen, M. W. Larsson, L. E. Fröberg, and L. Samuelson, Phys. Rev. B 72, 201307(R) (2005).

${ }^{24}$ H. A. Nilsson, C. Thelander, L. E. Fröberg, J. B. Wagner, and L. Samuelson, Appl. Phys. Lett. 89, 163101 (2006).

25 C. Fasth, A. Fuhrer, L. Samuelson, V. N. Golovach, and D. Loss, Phys. Rev. Lett. 98, 266801 (2007).

26 B. J. Ohlsson, M. T. Björk, M. H. Magnusson, K. Deppert, L. Samuelson, and L. R. Wallenberg, Appl. Phys. Lett. 79, 3335 (2001).

27 H. Shtrikman, R. Popovitz-Biro, A. Krctinin, and M. Heiblum, Nano Lett. 9, 215 (2009).

28 C. Galland, A. Högele, H. E. Türeci, and A. Imamoğlu, Phys. Rev. Lett. 101, 067402 (2008).

${ }^{29}$ G. Lindwall, A. Wacker, C. Weber, and A. Knorr, Phys. Rev. Lett. 99, 087401 (2007).

30 C. Weber, A. Fuhrer, C. Fasth, G. Lindwall, L. Samuelson, and A. Wacker, Phys. Rev. Lett. 104, 036801 (2010).

31 Y. Yin and M. W. Wu, J. Appl. Phys. 108, 063711 (2010).

32 M. Trif, V. N. Golovach, and D. Loss, Phys. Rev. B 77, 045434 (2008).

33 C. L. Romano, P. I. Tamborenea, and S. E. Ulloa, Physica E, 41, 1577 (2009).

34 B. A. Auld, Acoustic Fields and Waves in Solids (Willey,
New York, 1973).

35 T. Takagahara, Phys. Rev. Lett. 71, 3577 (1993).

36 M. A. Stroscio, K. W. Kim, S. Yu, and A. Ballato, J. Appl. Phys. 76, 4670 (1994).

37 S. Yu, K. W. Kim, M. A. Stroscio, and G. J. Iafrate, Phys. Rev. B 51, 4695 (1995).

38 S. M. Komirenko, K. W. Kim, M. A. Stroscio, and V. A. Kochelap, Phys. Rev. B 58, 16360 (1998).

39 H. Suzuura and T. Ando, Phys. Rev. B 65, 235412 (2002).

40 A. N. Cleland, Foundation of Nanomechanics (SpringerVerlag, Berlin, 2003).

41 P.-M. Chassaing, F. Demangeot, N. Combe, L. SaintMacary, M. L. Kahn, and B. Chaudret, Phys. Rev. B 79, 155314 (2009).

42 C. Weber, G. Lindwall, and A. Wacker, Phys. Status Solidi B 246, 337 (2009).

43 A. D. Bykhovski, V. V. Kaminski, M. S. Shur, Q. C. Chen, and M. A. Khan, Appl. Phys. Lett. 68, 818 (1996).

44 N. Nishiguchi, Phys. Rev. B 54, 1494 (1996).

45 Y. Ohno, D. K. Young, B. Beschoten, F. Matsukura, H. Ohno, and D. D. Awschalom, Nature 402, 790 (1999).

${ }^{46}$ W. Löffler, D. Tröndle, J. Fallert, H. Kalt, D. Litvinov, D. Gerthsen, J. Lupaca-Schomber, T. Passow, B. Daniel, J. Kvietkova, M. Grün, C. Klingshirn, and M. Hetterich, Appl. Phys. Lett. 88, 062105 (2006).

47 A. Shabaev, A. L. Efros, D. Gammon, and I. A. Merkulov, Phys. Rev. B 68, 201305(R) (2003).

48 M. Atatüre, J. Dreiser, A. Badolato, A. Högele, K. Karrai, and A. Imamoğlu, Science 312, 551 (2006).

49 D. Press, T. D. Ladd, B. Zhang, and Y. Yamamoto, Nature 456, 218 (2008).

50 Optical Orientation, edited by F. Meier and B. P. Zakharchenya (North-Holland, Amsterdam, 1984).

51 A. Pfund, I. Shorubalko, K. Enssilin, and R. Leturcq, Phys. Rev. B 79, 121306(R) (2009).

52 Semiconductors, Landolt-Börnstein, edited by O. Madclung, Vol. 17a, (Springer-Verlag, Berlin, 1987).

53 A. E. Hansen, M. T. Björk, C. Fasth, C. Thelander, and L. Samuelson, Phys. Rev. B 71, 205328 (2005).

${ }^{54}$ V. N. Golovach, A. Khaetskii, and D. Loss, Phys. Rev. B 77, 045328 (2008). 